\title{
AN ELEMENTARY PROOF OF THE JORDAN-SCHOENFLIES THEOREM ${ }^{1}$
}

\author{
STEWART S. CAIRNS
}

\section{Introduction.}

The jordan-schoenflies theorem. A simple closed curve $c$ in a plane $E$ separates $E$ into two regions. There exists a self-homeomorphism of $E$ under which $c$ is mapped onto a circle.

The exterior of a bounded closed point set $b$ in $E$ will mean the unbounded region of the complementary set $E-b$. The remainder of $E-b$, if not vacuous, will be called the interior of $b$.

(A) As a corollary to the above theorem, $c$ is intersected by any simple arc with one end point interior and one exterior to $c$.

This paper contains an elementary constructive proof of the Jordan-Schoenflies Theorem, motivated by the belief that such a proof should be presented at a fairly early stage to students of topology and analysis. To that end, it is desirable that the argument be disassociated from conformal mapping theory and be accomplished by methods as elementary as possible.

2. Preliminary results. Let $(x, y)$ denote a rectangular cartesian coordinate system in $E$. The following two statements can be quickly established by familiar methods.

(A) Let $b_{1}$ and $b_{2}$ denote two simple closed curves for each of which the Jordan-Schoenflies Theorem holds. Then an arbitrary homeomorphism between $b_{1}$ and $b_{2}$ can be extended to a self-homeomorphism of E.

(B) If the Jordan-Schoenflies Theorem holds for $b_{1}$ and $b_{2}$, and if the intersection $b_{1} \cdot b_{2}$ is a simple arc $b$, then the Jordan-Schoenflies Theorem holds for the simple closed curve $b_{1}+b_{2}-b^{\prime}$, where $b^{\prime}$ denotes $b$ without its end points.

Theorem 2.1. The Jordan-Schoenflies Theorem holds for a simple closed polygon $p$. A polygonal path crossing $p$ at just one point and otherwise not meeting $p$ has one end point exterior and one interior to $p$.

Proof. (C) The result offers no difficulty when $p$ is a triangle.

Presented to the Society, December 30, 1948; received by the editors June 5, 1950 and, in revised form, January $15,1951$.

${ }^{1}$ The proof has been substantially shortened and simplified since the presentation of the paper to the Society as a result of suggestions by Mr. John Nash of Princeton University. 
Suppose $p$ has $n>3$ vertices and assume Theorem 2.1 for all polygons having fewer than $n$ vertices.

Let $\alpha$ be the set of all points each attainable from the exterior of $p$ by a polygonal path crossing $p$ at just one point and otherwise not meeting $p$.

Lемма 2.1. There exists a line segment d joining two vertices of $p$ on $\alpha$.

To establish Lemma 2.1 , let $P_{0}$ be the point on $p$ with the smallest ordinate among those where the absicssa is smallest. Then $P_{0}$ is a vertex of $p$. Let $P_{1}, P_{2}$ be the vertices consecutive with $P_{0}$ in either sense along $p$. Let $\delta$ denote the triangular region $P_{0} P_{1} P_{2}$. Then either $P_{1} P_{2}$ satisfies Lemma 2.1 or else $\bar{\delta}$ contains vertices of $p$ other than $\left(P_{1}, P_{2}\right)$. In the latter case, $P_{0} P_{3}$ satisfies the lemma if $P_{3}$ is one of the vertices on $\bar{\delta}-\left(P_{1}, P_{2}\right)$ with least abscissa greater than the abscissa of $P_{0}$.

Let $p_{1}, p_{2}$ be the two polygonal arcs into which the end points of $d$ divide $p$. Then the hypothesis of the recurrency (see (A) above) applies to $p_{1}+d$ and to $p_{2}+d$. Theorem 2.1 now follows for $p$, and hence follows in general, with the aid of result (B).

\section{Approximation to a sector.}

LEMмA 3.1. Let $c$ be a Jordan curve with at least one interior point $P$ and let $\alpha$ be the maximal region of $E-c$ containing $P$. Then any chord

$$
d=D_{1} D_{2}
$$

of $c$ on $\alpha$ separates $\alpha$ into two regions.

Proof. Let $c_{1}, c_{2}$ be the two arcs into which $D_{1}, D_{2}$ separate $c$. Let $p$ denote an arbitrary simple closed polygon crossing $d$ at just one point $M$, and not meeting $d$ elsewhere.

(A) The polygon $p$ intersects $c_{i}(i=1,2)$.

This auxiliary result follows from the facts that (1) $p$ separates $D_{1}$ from $D_{2}$, by Theorem 2.1 , and (2) $c_{i}$ joins $D_{1}$ and $D_{2}$.

(B) Let $p$ be traced from $M$ in either sense to the first points encountered on $c$. This leads to two distinct points, $P_{1}$ and $P_{2}$, on $c_{1}$ and $c_{2}$ respectively.

To establish (B), let $p_{0}$ be the arc $P_{1} M P_{2}$ of $p$. Suppose that (B) is false and that both end points of $p_{0}$ are on $c_{1}$, for example. Let $c_{0}$ be the arc of $c_{1}$ which they bound. From parts of $p_{0}$ and a suitable

${ }^{2}$ A simple arc will be said to join its end points on a region if the entire arc, save perhaps for either or both end points, is on that region. 
polygonal approximation to $c_{0}$, it is possible to put together a simple closed polygon through $M$, meeting $\left(c_{2}+d\right)$ only at $M$, where it crosses $d$. By the argument for (A), this is contradictory, since such a polygon would necessarily meet $c_{2}$.

(C) Let $p_{i}$ be the arc of $p_{0}$ with $M$ and $P_{i}$ for end points $(i=1,2)$, and let $\alpha_{i}$ be the set of all points which can be joined to $p_{i}$ by arcs not meeting $c+d$. Then (1) $\alpha_{1}$ and $\alpha_{2}$ are disjoint and (2) $\bar{\alpha}_{1}+\bar{\alpha}_{2}=\bar{\alpha}$.

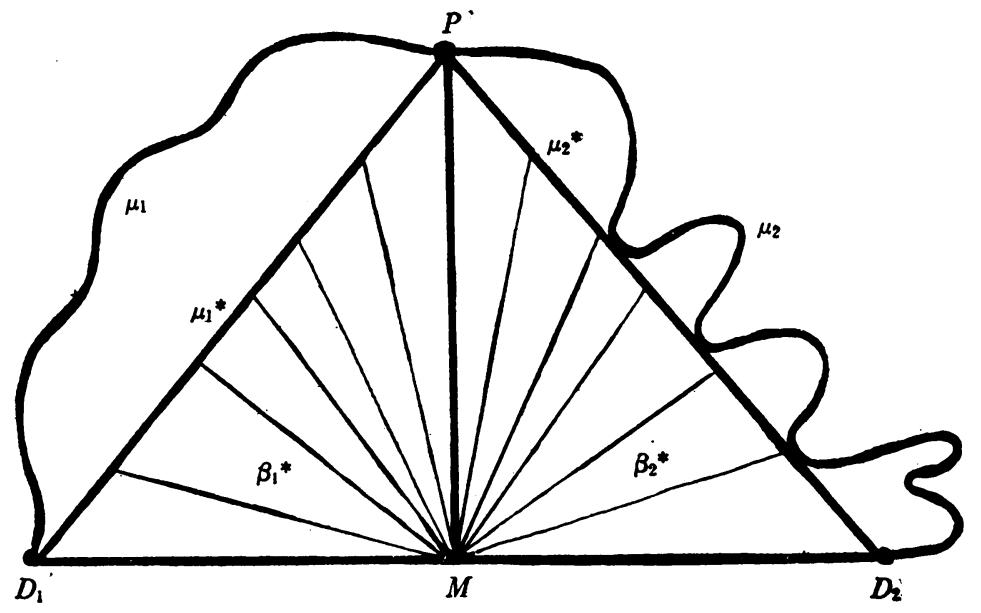

Figure 3.1

If $\alpha_{1}$ and $\alpha_{2}$ were not disjoint, then any point common to them could be joined to $p_{i}(i=1,2)$ by a polygonal arc $q_{i}$ on $\alpha_{i}$. From parts of $p_{1}, p_{2}, q_{1}$, and $g_{2}$, a polygon could be put together, leading to the same sort of contradiction as in the argument for (B). Part (2) of (C) presents no difficulty. The lemma and the corollary below now follow at once.

COROLLARY. In the above notation, the boundary of $\alpha_{i}$ is on $d+c_{i}$ $(i=1,2)$.

Either of the two parts into which a chord $d=D_{1} D_{2}$ separates $\alpha$ will be called a sector $\beta$ of $\alpha$. As a preliminary to proving that $\alpha$ is a 2-cell, a method will now be developed for partially filling in $\beta$ by an approximating region $\beta^{*}$. In accordance with the preceding corollary, the boundary of $\beta$ is on $d+\mu$, where $\mu$ is one of the arcs of $c$ with $D_{1}$ and $D_{2}$ for end points. From the midpoint $M$ of $d$, let a ray normal to $d$ be extended into $\beta$, and let $P$ be the first point of $c$ on that ray. Then, by the above corollary, $P$ is on $\mu$ [see Fig. 3.1]. Let $\mu_{i}$ be the arc of $\mu$ with $P$ and $D_{i}$ for end points $(i=1,2)$. By 
Lemma 3.1 and the corollary, $M P$ separates $\beta$ into two regions $\beta_{i}(i=1,2)$, where the boundary of $\beta_{i}$ is on $\mu_{i}+M D_{i}+M P$. Let $\delta_{i}$ be the interior of the triangle $M P D_{i}$. A subset $\beta_{i}^{*}$ of $\delta_{i}$ will next be defined, as an approximation to $\beta_{i}$. Its boundary will be the union of $M P, M D_{i}$, and an arc $\mu_{i}^{*}$ joining $D_{i}$ and $P$ on $\bar{\delta}_{i}$.

Case I. $\left(\delta_{i} \subset \beta_{i}\right)$. In this case $\mu_{i}^{*}=P D_{i}$ and $\beta_{i}^{*}=\delta_{i}$ (see $\beta_{1}^{*}$ in Fig. 3.1).

Case II. $\left(\delta_{i} \nsubseteq \beta_{i}\right)$. In this case, let $\mu_{i}^{\prime}$ be the intersection of $\mu_{i}$ with $\delta_{i}$. The convex hull of $\mu_{i}^{\prime}+P D_{i}$ is then bounded by a convex closed curve $\mu_{i}^{\prime}+D_{i} P$; and the arc $\mu_{i}^{*}$ separates $\delta_{i}$ into two regions, of which the one with $M$ on its boundary will be $\beta_{i}^{*}$ [see $\beta_{2}^{*}$ in Fig. 3.1].

(D) The approximation $\beta^{*}$ to $\beta$ is now defined as the union of $\beta_{1}^{*}, \beta_{2}^{*}$ and the open segment $M P$. It is uniquely determined by $\beta$.

(E) The arc

$$
\mu^{*}=\mu_{1}^{*}+\mu_{2}^{*}
$$

is the union of a subset $c^{*}$ of $\mu$ and a denumerable set of chords of $c$. As a point $P^{*}$ traces $\mu^{*}$ from $D_{1}$ to $D_{2}$, the open segment $M P^{*}$ sweeps out the entire region $\beta^{*}$.

4. An interior region of a Jordan curve. Under the hypotheses of Lemma 3.1, let $h(c)$ be an arbitrary but fixed homeomorphic mapping of $c$ onto a circle $k$. The images of $c^{*}, \mu$, and $D_{i}$ [see $\$ 3$ for notation] will be denoted by

$$
\begin{aligned}
k^{*} & =h\left(c^{*}\right), \\
v & =h(\mu), \\
E_{i} & =h\left(D_{i}\right) \quad(i=1,2) .
\end{aligned}
$$

Then $v$ is an arc of $k$ with end points $E_{1}, E_{2}$, and $k^{*}$ is a subset of $v$. Let $h(c)$ now be extended to each chord of $c$ on $\mu^{*}$ [see $\left.\$ 3(\mathrm{E})\right]$ and to the chord $d$ by the requirement that these cords map linearly onto chords of $k$. This extends $h(c)$ into a map $h\left(c+d+\mu^{*}\right)$. Let the images of $d$ and $\mu^{*}$ [see $\S 3$ ] be

$$
\begin{aligned}
e & =h(d), \\
v^{*} & =h\left(\mu^{*}\right) .
\end{aligned}
$$

Then $e$ is the chord $E_{1} E_{2}$ and $v^{*}$ is a simple arc from $E_{1}$ to $E_{2}$ on the closure of the region $\gamma$ bounded by $e+v$. The arc $v^{*}$ is the union of $k^{*}$ and a denumerable set of chords of $k$.

The image $N=h(M)$ of $M$ is the midpoint of $e$. As $P^{*}$ traces $\mu^{*}$ from $D_{1}$ to $D_{2}$, its image $Q^{*}=h\left(P^{*}\right)$ traces $v^{*}$ from $E_{1}$ to $E_{2}$, and the 
open segment $N Q^{*}$ sweeps out an approximation $\gamma^{*}$ to $\gamma$, bounded by $e+v^{*}$. Let $h$ now be extended over $\beta^{*}$ by the requirement that it map each segment $M P^{*}$ linearly onto the segment $N Q^{*}$.

(A) This completes the extension of $h(c)$ into a homeomorphic mapping $h\left(c+\beta^{*}\right)$ of $c+\beta^{*}$ onto $k+\gamma^{*}$. The extension is uniquely determined, given $h(c)$ and the sector $\beta$.

THEOREM 4.1. Under the hypotheses of Lemma 3.1, an arbitrary homeomorphic mapping $h(c)$ of $c$ onto a circle $k$ can be extended into a homeomorphic mapping $H(\bar{\alpha})$ of $\bar{\alpha}$ onto $\bar{K}$, where $K$ is the interior of $k$.

Let $d=D_{1} D_{2}$ be an arbitrary chord of $c$ on $\alpha$. Let $\beta^{*}, \gamma^{*}$ be approximations, as defined in $\$ 3(\mathrm{D})$, to the two sectors into which $d$ separates $\alpha$. Let $\alpha_{1}$ denote the union of $\beta^{*}, \gamma^{*}$ and the open segment $D_{1} D_{2}$.

(B) By the extension process of statement (A), let $h(c)$ be extended over both $\bar{\beta}^{*}$ and $\bar{\gamma}^{*}$, hence over $\bar{\alpha}_{1}$. This defines a homeomorphic mapping $H_{1}\left(\bar{\alpha}_{1}+c\right)$ of $\bar{\alpha}_{1}+c$ onto a certain subset $\bar{K}_{1}+k$ of $\bar{K}$.

The definition of $H_{1}\left(\bar{\alpha}_{1}+c\right)$ is the first step of a recurrent process, based on the following hypothesis, which is easy to verify for $j=1$.

Hyротнesis. For some positive integer $j$, the sets $\alpha_{i}, K_{i}$ and the homeomorphisms $H_{i}(i=1,2, \cdots, j)$ have been so defined that:

(1) The domain of $H_{i}$ is $\bar{\alpha}_{i}+c$, where $\alpha_{i}$ is a 2-cell on $\alpha$.

(2) The boundary of $\alpha_{i}$ is a simple closed curve $c_{i}$ which is the union of a point set $c_{i}^{*}$ on $c$ and a denumerable set of chords of $c$ on $\alpha$.

(3) The image $k_{i}=H_{i}\left(c_{i}\right)$ is the union of a subset $k_{i}^{*}=H_{i}\left(c_{i}^{*}\right)$ of $k$ and a denumerable set of chords of $k$.

(4) The mapping $H_{i}$ is linear between the chords of $c$ on $c_{i}$ and the chords of $k$ on $k_{i}$.

(5) Each map $H_{i+1}$ is an extension of $H_{i}$ and of $h(c)$.

Let $d_{i}(i=1,2, \cdots)$ denote the chords of $c$ on $c_{j}$. Of the two sectors into which $d_{i}$ separates $\alpha$ (see Lemma 3.1), let $\beta_{i}$ be the one which contains no point of $\alpha_{j}$, and let $\beta_{i}^{*}$ be the approximation to $\beta_{i}$ defined in $\$ 3(D)$.

(C) The region $\alpha_{j+1}$ is now defined as

$$
\alpha_{j+1}=\alpha_{j}+\sum \beta_{i}^{*}+\sum d_{i}^{\prime}
$$

where $d_{i}^{\prime}$ is the chord $d_{i}$ without its end points. The homeomorphism $H_{j+1}$ is now defined on $\bar{\alpha}_{j+1}-\bar{\alpha}_{j}$ by extending $h(c)$ over each $\bar{\beta}_{*}^{x}$, using the process of (A) above. On $c_{j}$, this extension agrees with $H_{j}$, as a consequence of the linearity requirements in the extension process and in part (4) of the above hypothesis. 
On $\bar{\alpha}_{j}$, let $H_{j+1}$ be defined as identical with $H_{j}$. It is then easy to verify the above Hypothesis with $j+1$ in place of $j$ where

$$
\begin{aligned}
K_{j+1} & =H_{j+1}\left(\alpha_{j+1}\right), \\
k_{j+1} & =H_{j+1}\left(c_{j+1}\right) .
\end{aligned}
$$

LemMA 4.1. For any $\epsilon>0$, there exists an integer $j$ so large that every circular region of radius $\epsilon$ about a point on contains a sector on $\alpha$ outside $\alpha_{j}$ and cut off by a chord of $c_{j}$. In other words, the regions of Fig. 3.1 become uniformly small as $j$ increases.

Suppose the lemma false. As a consequence of the recurrent process for defining the regions $\alpha_{j}$, it follows that, for some $\epsilon>0$, there exists at least one circular neighborhood of radius $\epsilon$ with center on $c$ containing no point of $\sum c_{i}^{*}$.

Let $c_{0}$ be a maximal arc of $c-\sum c_{t}^{*}$, and let $P_{0}, P_{0}^{\prime}$ be its end points . Let $N, N^{\prime}$ be the circular neighborhoods about $P_{0}, P_{0}^{\prime}$ respectively each of radius $d\left(P_{0}, P_{0}^{\prime}\right) / 3$. By definition of $c_{0}$, it is possible to find two points $\left(D_{1}, D_{2}\right)$ such that (1) $D_{1}$ and $D_{2}$ are the end points of a chord $d$ of $c$ on the curve $c_{i}$ [see Hypothesis, Part (2)] for some value of $i$. (2) If $c_{i}^{\prime}$ is the arc of $c$ which has $D_{1}, D_{2}$ for end points and contains $c_{0}$, then $\left(c_{i}^{\prime}-c_{0}\right) \subset\left(N+N^{\prime}\right)$. (3) $D_{1}$ and $D_{2}$ are so close to $P_{0}$ and $P_{0}^{\prime}$ respectively, that the perpendicular bisector $n$ of $d$ does not meet $\bar{N}+\bar{N}^{\prime}$. In the extension process, a point of $c_{i+1}^{*}$ is common to $n$ and $c_{i}^{\prime}$, hence is on the arc $c_{0}$. Since this contradicts the definition of $c_{0}$, Lemma 4.1 is proved.

Corollary. Every point of $\alpha$ is on one of the 2-cells $\alpha_{i}$.

Assume the contrary, and let $Q$ be a point on $\alpha-\sum \alpha_{i}$. For each value of $i$, there is a chord $d_{i}$ on $c_{i}$ which separates $\alpha$ into two sectors, one of which, $\beta_{i}$, contains $Q$, while the other contains $\alpha_{i}$. Let $e_{i}$ be the arc of $c$ on $\bar{\beta}_{i}$. Then, as a consequence of Lemma 4.1, there is just one point $Q^{*}$ common to all the arcs $e_{i}$. For $i$ large enough, any given neighborhood $N\left(Q^{*}\right)$ will contain $d_{i}+c_{i}$ and hence $\beta_{i}$. Since $N\left(Q^{*}\right)$ need not contain $Q$, the corollary follows.

Now let $H$ be defined as the common extension of all the homeomorphisms $H_{i}$. By the above corollary, the domain of $H$ is $\alpha+c$. Furthermore, $H$ is continuous on $\bar{\alpha}$. Its continuity on $\alpha$ follows from the continuity of the $H_{i}$, while its continuity at points on $c$ follows from Lemma 4.1. Hence the mapping $H$ fulfills the requirements of Theorem 4.1.

Let $b_{1}$ and $b_{2}$ denote two Jordan curves, each with an interior, and let $\beta_{i}$ be a 2 -cell with $b_{i}$ for boundary $(i=1,2)$, in accordance with 
Theorem 4.1. Suppose the intersection of $b_{1}$ and $b_{2}$ is an arc $b$, where $b_{1}-b$ is exterior to $b_{2}$ and $b_{2}-b$ is exterior to $b_{1}$. Let $b^{\prime}$ denote $b$ without its end points, and let

$$
\beta=\beta_{1}+b^{\prime}+\beta_{2} .
$$

(D) As a corollary to Theorem $4.1, \beta$ is a 2-cell with $b_{1}+b_{2}-b^{\prime}$ for boundary, and any homeomorphism between this boundary and a circle $k$ can be extended into a homeomorphism between $\bar{\beta}$ and $\bar{K}$ (see Theorem 4.1 for notation). It will be said that $\beta$ is obtained by amalgamating $\beta_{1}$ and $\beta_{2}$ across b.

\section{Completion of the proof.}

LEMMA 5.1. Let $c$ satisfy the hypotheses of Lemma 3.1 and hence of Theorem 4.1. Let $g$ be a simple arc joining two distinct points $P_{1}$ and $P_{2}$ of $c$ and lying on $\alpha$, save for $P_{1}, P_{2}$. Let $c_{1}, c_{2}$ be the two arcs into which $P_{1}, P_{2}$ divide $c$. Then $g$ separates $\alpha$ into two 2-cells $\alpha_{1}, \alpha_{2}$ where $\alpha_{i}$ has $g+c_{i}$ for boundary $(i=1,2)$.

Proof. By Theorem 4.1, the lemma reduces to the case where $c$ is a circle and $\alpha$ is its interior. If $g$ did not separate $\alpha$, a polygonal arc $p$ could be constructed joining $c_{1}$ to $c_{2}$ on $\alpha$ without meeting $g$. This arc $p$ could be completed outside $c$ to a simple closed polygon. By Theorem 2.1, using an arc of circle instead of a polygonal path, such a polygon must separate $P_{1}$ from $P_{2}$ and hence must intersect $g$, contrary to its definition. It follows that $g+c_{i}$ satisfies the hypotheses of Theorem 4.1. Let $\alpha_{i}$ be a 2-cell bounded by $g+c_{i}$ in accordance with that theorem. By $\S 4(\mathrm{D}), \alpha_{1}$ and $\alpha_{2}$ can be amalgamated across $g$ to obtain the 2-cell, $\alpha$, bounded by $c$.

Next consider an arbitrary simple closed curve $c$. Let $p$ be a simple closed polygon, meeting $c$ in just two points, $P_{1}$ and $P_{2}$, and otherwise exterior to $c$. Such a polygon is easy to define, if $P_{1}$ and $P_{2}$ are chosen as points of maximum and minimum ordinates, respectively, on $c$.

Let $c_{1}$ and $c_{2}$ be the two arcs into which $P_{1}$ and $P_{2}$ divide $c$. As a consequence of Theorem 2.1 and Lemma 5.1, $c_{i}$ separates the interior, $\rho$, of $p$ into two 2-cells $\alpha_{i}$ and $\beta_{i}$, one of which, $\beta_{i}$, contains $c_{j}$ $(i=1, j=2)$ and $(i=2, j=1)$. Similarly, $c_{1}$ separates $\beta_{2}$ into two 2 -cells, one of which, $\alpha$, has $c_{1}+c_{2}$ for boundary. Any point on $\alpha$ is interior to $c$, since any arc joining it to $p$ must meet either $c_{1}$ or $c_{2}$. This establishes the following result.

LEMMA 5.2. Any Jordan curve has an interior.

Now let $\alpha_{1}$ and $\alpha$ be amalgamated across $c_{1}$ [see $\left.\S 4(\mathrm{D})\right]$ and let 
the resulting 2-cell be amalgamated with $\alpha_{2}$ across $c_{2}$ to obtain a 2 -cell $\rho$ with $p$ for boundary. By Theorem 2.1, $\rho$ is the interior of $p$. Since $\alpha_{1}$ and $\alpha_{2}$ are exterior to $c$, the 2-cell $\alpha$ constitutes the entire interior of $c$. The Jordan-Schoenflies Theorem now follows readily in all its generality.

UNIVERSITY OF ILLINOIS

\section{A NOTE ON CURVATURE AND BETTI NUMBERS}

\section{H. GUGGENHEIMER}

1. S. Bochner has proved the following theorem $[2]:{ }^{1}$ Let $M^{(m)}$ be a closed manifold with complex structure $[4 ; 7]$ of complex dimension $m$, on which there exists a Kähler-metric $[2 ; 3 ; 5]^{2}$

$$
\begin{aligned}
d s^{2} & =g_{i k^{*}}\left(d z^{i} d z^{*}\right),^{3} \\
\frac{\partial g_{i k^{*}}}{\partial z_{l}} & =\frac{\partial g_{l k^{*}}}{\partial z_{i}} .
\end{aligned}
$$

Let $R_{i k^{*}}$ denote the Ricci tensor and

$$
P_{h i^{*} j k^{*}}=R_{h i^{*} j k^{*}}-\frac{1}{m+1}\left(g_{h i^{*}} R_{j k^{*}}+g_{h k^{*}} R_{i^{*} j}\right)
$$

the tensor of projective curvature. In every point of $M^{(m)}$ we form the numbers

$$
\begin{aligned}
& L=\inf _{\xi} \frac{-R_{i k^{*}} \xi^{i \xi k^{*}}}{\xi^{i} \xi_{i}}, \\
& P=\sup _{\xi}\left|\frac{P_{h i^{*} j k^{*} \xi^{h i^{*}} \xi^{j k^{*}}}}{\xi^{h i^{*}} \xi_{h i^{*}}}\right|,
\end{aligned}
$$

with all vectors $\xi^{i}$ and skew-symmetric tensors $\xi^{i j^{*}}$ attached to the point in question. If

$$
L>0
$$

Received by the editors December 8, 1950.

${ }^{1}$ Numbers in brackets refer to the bibliography at the end of the paper.

2 Products of differentials in parentheses denote ordinary products, products without parentheses are skew products.

${ }^{3}$ We denote by $i^{*}$ the index relative to $z^{* i}$. 